\title{
Perceptions of molecular epidemiology studies of HIV among stakeholders
}

\author{
Cynthia Schairer, ${ }^{1}$ Sanjay R. Mehta, ${ }^{1-3}$ Staal A. Vinterbo, ${ }^{1}$ Martin Hoenigl, \\ Michael Kalichman, ${ }^{3}$ Susan Little ${ }^{1}$ \\ ${ }^{1}$ Department of Medicine, University of California San Diego, CA; ${ }^{2}$ Department of Medicine, San \\ Diego Veterans Affairs Medical Center, San Diego, CA; ${ }^{3}$ Department of Pathology, University of \\ California San Diego, CA, USA
}

\begin{abstract}
Significance for public health
When molecular epidemiology is used to study HIV, it can demonstrate how HIV infections are related and how to target prevention efforts. Applying these analyses for maximal benefit in the fight against HIV would almost certainly make individuals whose data are analyzed vulnerable to discovery. However, absolute protection of this sensitive information would require that research into these methods not be done. The success of HIV molecular epidemiology will depend on finding a balance between public health and the interests of individuals living with HIV. The stakeholders interviewed in this study agreed that molecular epidemiology should be used to study HIV epidemics and transmission despite risks to privacy. However, these interviews also highlighted the difficulty of understanding molecular epidemiology and its privacy implications. For HIV molecular epidemiology to continue, privacy protections must go beyond simply masking traditional identifiers and assuming participants are informed enough to consent to the risks.
\end{abstract}

\section{Abstract}

Background: Advances in viral sequence analysis make it possible to track the spread of infectious pathogens, such as HIV, within a population. When used to study HIV, these analyses (i.e., molecular epidemiology) potentially allow inference of the identity of individual research subjects. Current privacy standards are likely insufficient for this type of public health research. To address this challenge, it will be important to understand how stakeholders feel about the benefits and risks of such research.

Design and Methods: To better understand perceived benefits and risks of these research methods, in-depth qualitative interviews were conducted with HIV-infected individuals, individuals at high-risk for contracting HIV, and professionals in HIV care and prevention. To gather additional perspectives, attendees to a public lecture on molecular epidemiology were asked to complete an informal questionnaire.

Results: Among those interviewed and polled, there was near unanimous support for using molecular epidemiology to study HIV. Questionnaires showed strong agreement about benefits of molecular epidemiology, but diverse attitudes regarding risks. Interviewees acknowledged several risks, including privacy breaches and provocation of anti-gay sentiment. The interviews also demonstrated a possibility that misunderstandings about molecular epidemiology may affect how risks and benefits are evaluated.

Conclusions: While nearly all study participants agree that the benefits of HIV molecular epidemiology outweigh the risks, concerns about privacy must be addressed to ensure continued trust in research institutions and willingness to participate in research.

\section{Introduction}

Despite rapid advances in treatment and prevention of HIV, rates of new infections remain unacceptably high. ${ }^{1,2}$ Targeting limited resources to the highest risk individuals and groups (social networks) remains a major challenge of prevention efforts. Molecular epidemiology (ME) offers an approach to understand patterns of transmission. ME takes advantage of the fact that many RNA viruses, like HIV, evolve at a rapid pace. ${ }^{3}$ This leads to the development of significant viral diversity within infected individuals, and across populations over time. A virus transmitted recently from one person to several others will result in a cluster (network) of individuals with more closely related viruses. Thus, viral sequence relationships within the HIV infected population can be exploited using ME to not only reconstruct the evolutionary history of the virus but to infer patterns of transmission within a sampled population. On a local level, aggregation and integration of molecular, clinical and demographic data offers a unique opportunity to better understand the dynamics of local transmission networks. ${ }^{4-6}$ Inferences made on the spread of HIV in a local sub-epidemic could then potentially be used to identify key sub-populations with high rates of transmission to better target prevention and treatment resources. For example, HIV ME has been used to identify sociodemographic and geographic hotspots of HIV transmission. ${ }^{7}$

Molecular epidemiology is a powerful tool in the study of infectious diseases, but is little known outside the scientific communities that use it. When applied to the study of HIV transmission ME can provide researchers with important insights into the spread of HIV and also identify methods to interrupt this spread. However, this technique also has the potential to generate extremely sensitive information and allow inference of the identity of individual participants, presenting unique challenges for maintaining privacy for research participants while maximizing the utility of the information produced. Without sufficient protections, ME data could, for example, be used to implicate individual research participants in the spread of HIV ${ }^{8,9}$

The more information collected about an individual, the easier it is to deduce the identity of that person without relying on traditional categories of identifying information such as name, address, or social security number. This was demonstrated by Gymrek et $a l .{ }^{10}$ in a 2013 study, where public databases were used to triangulate the identifiers of participants in public sequencing projects, enabling identification of some individuals who participated. In the context of HIV ME research, most scientific journals require that the analysed sequence data be published, information that may be unique to each individual. Coupled with the fact that the study populations are often small, these factors can significantly increase the risk of identification, ${ }^{11}$ and may suggest, but never 
definitively prove, possible sources of transmission. ${ }^{8,9}$ Furthermore, this information may be used by public health departments in outbreak investigations, and the published reports of these investigations, even when devoid of protected health information, may still provide clues to the identities of individuals involved. ${ }^{12}$ With respect to the analyses of HIV genetic data to infer transmission patterns, the stakes are higher as 33 states in the US have laws that criminalize the transmission of HIV..$^{13,14}$ Identification of an individual in an HIV transmission network could suggest, although not prove, high risk behaviour such as sharing needles or sex with a large number of partners. Though these data do not exclude the presence of one or more un-sampled infected individuals in the transmission chain, genetic similarities between infections may be misinterpreted as evidence of direct contact with other individuals. Because HIV ME data could implicate an individual in the transmission of HIV, a breach of privacy could result in devastating personal and legal consequences.

HIV ME researchers must protect personal privacy if they are to maintain trust with the populations they study. They must strike a balance that reasonably protects individuals' privacy, but still allows them to report meaningful scientific findings and bring the power of HIV ME to the fight against HIV. The right balance will honour the interests of the research subjects, the researchers, professionals in HIV care and prevention, and the public. However, determining these interests is difficult when so few people have adequate knowledge and understanding of how molecular epidemiology works. To meet these challenges, we previously proposed a strategy for handling data generated by HIV ME using techniques that allow formal quantitative tradeoff between privacy and accuracy of information extracted from the data. ${ }^{11}$ The success of such techniques requires making tradeoffs among the complex interests of researchers, research participants, HIV care providers, and the public. This study is a first attempt to assess how an optimal tradeoff might be found by asking how stakeholders weigh the benefits and risks involved in HIV ME. We conducted in-depth qualitative interviews with individuals living with HIV, individuals at risk for acquiring HIV, and professionals working in HIV care and prevention. We also distributed an informal questionnaire to the audience of a public lecture on the topic of HIV ME. In this paper, we discuss how our research participants responded to an explanation of HIV ME, the risks they anticipated and reported being most concerned about, and the common misunderstandings of HIV ME revealed in the interviews.

\section{Materials and Methods}

\section{Ethical approval}

All procedures performed in studies involving human participants were in accordance with the ethical standards of the University of California San Diego Human Research Protections program (UCSD HRPP). Informed consent was obtained from all individual participants included in the study, except where a waiver of consent for anonymous questionnaires was obtained from the UCSD HRPP.

\section{Interviews}

Qualitative interviews were conducted with forty individuals over six months beginning in February of 2015. Four categories of stakeholders were recruited: HIV infected individuals $(n=11)$ and HIV uninfected but at-risk individuals $(n=10)$, medical professionals (doctors and nurses working with HIV patients) $(n=11)$, and non-medical professionals working in HIV prevention and care (i.e. activists, non-profit administrators, epidemiologists) $(\mathrm{n}=8)$. Interviewees were recruited through the research and professional networks of SL and SM in and around San Diego, California. Many HIV positive and HIV at risk recruits were concurrently enrolled in on-going HIV prevention studies. Table 1 summarizes demographic characteristics of the four groups.

Two versions of an interview guide were piloted and revised

Table 1. Demographic summary of groups interviewed.

\begin{tabular}{|c|c|c|c|c|c|}
\hline & $\mathrm{HIV+}$ & At risk for HIV infection & Medical professional & Non-medical professional & All \\
\hline Number & 11 & 10 & 11 & 8 & 40 \\
\hline $\begin{array}{l}\text { Gender } \\
\text { Men } \\
\text { Women } \\
\end{array}$ & $\begin{array}{l}9 \\
2\end{array}$ & $\begin{array}{c}10 \\
0\end{array}$ & $\begin{array}{l}7 \\
4\end{array}$ & $\begin{array}{l}2 \\
6\end{array}$ & $\begin{array}{l}28 \\
12\end{array}$ \\
\hline $\begin{array}{l}\text { Sexuality } \\
\text { Men who have sex with men } \\
\text { Heterosexual } \\
\text { Sexuality not reported }\end{array}$ & $\begin{array}{l}7 \\
4 \\
0\end{array}$ & $\begin{array}{c}10 \\
0 \\
0\end{array}$ & $\begin{array}{l}4 \\
0 \\
7\end{array}$ & $\begin{array}{l}2 \\
0 \\
6\end{array}$ & $\begin{array}{c}23 \\
4 \\
13\end{array}$ \\
\hline Average age & 43 & 36 & 43 & 47 & 42.25 \\
\hline Age range & $23-65$ & $22-57$ & $31-64$ & $28-68$ & $22-68$ \\
\hline History of injection drug use & 4 & 0 & Not asked & Not asked & 4 \\
\hline $\begin{array}{l}\text { Race/Ethnicity } \\
\text { White } \\
\text { African American } \\
\text { Latino } \\
\text { Declined to answer }\end{array}$ & $\begin{array}{l}3 \\
2 \\
6 \\
0\end{array}$ & $\begin{array}{l}5 \\
1 \\
3 \\
1\end{array}$ & $\begin{array}{l}7 \\
1 \\
1 \\
0\end{array}$ & $\begin{array}{l}5 \\
0 \\
3 \\
0\end{array}$ & $\begin{array}{c}20 \\
4 \\
13 \\
1\end{array}$ \\
\hline $\begin{array}{l}\text { Education } \\
\text { Less than HS diploma } \\
\text { High school only } \\
\text { Some college } \\
\text { Bachelor's Degree } \\
\text { Some postgraduate } \\
\text { Postgraduate }\end{array}$ & $\begin{array}{l}3 \\
2 \\
4 \\
2 \\
0 \\
0\end{array}$ & $\begin{array}{l}1 \\
0 \\
2 \\
5 \\
2 \\
0\end{array}$ & $\begin{array}{c}0 \\
0 \\
0 \\
0 \\
0 \\
11^{*}\end{array}$ & $\begin{array}{l}0 \\
0 \\
0 \\
4 \\
0 \\
4\end{array}$ & $\begin{array}{c}4 \\
2 \\
6 \\
11 \\
2 \\
15\end{array}$ \\
\hline
\end{tabular}

*3 Registered Nurses, 8 Medical Doctors. 
before conducting the first interview. Pilot interviews were conducted with staff serving as mock interviewees. Each in-depth semi-structured interview lasted approximately one hour. All interviews were conducted by CS, a Ph.D. sociologist specializing in qualitative interviewing and observed by one other team member. CS had no formal training in molecular epidemiology or HIV prevention. Each interview followed a course unique to the interviewee, not all questions were asked of all respondents and some explanations and prompts were modified as we discovered the most effective wording.

All interviews were structured to move from a general explanation of molecular epidemiology to increasingly specific inquiries about potential privacy concerns. After collecting demographic information from the interviewee, the interviewer gave a basic explanation of molecular epidemiology (see Supplemental Material) using a mockup of an HIV transmission network map (Figure 1). This explanation was carefully crafted based on our experience in the public forum and with input from our team. CS also presented it to all professional interviewees and asked directly if they would change or add to it. As feedback was positive, the explanation was not substantially changed during the interviewing phase of the study.

After explaining HIV ME, the interviewer initiated a general discussion of questions and ideas offered by the interviewee about this explanation. Interviewees were then asked about various hypothetical scenarios illustrating ethical dilemmas related to molecular epidemiology. These scenarios were open-ended as they presented ethical dilemmas that have no determined resolution. For example, one scenario described a researcher identifying an outbreak of HIV at a university using HIV ME and asked the interviewee, Do you think the researchers would have an ethical obligation to act on this information? If so, what could they do?

The interviews offered many opportunities to explore stakeholders' understanding of and attitudes toward the potential risks of HIV ME. Most interviewees were asked about potential risks twice during the interview. After the initial explanation of HIV ME, they were asked to respond to open questions such as, What are your thoughts? or What do you think people could do with this? Some respondents immediately began to express concerns. In early interviews, some who did not raise concerns spontaneously were not asked about risks specifically. As the interview guide was refined, we began to ask specifically, Are there any risks to doing this research that come to mind? At the conclusion of the interview, respondents were again asked about the risks: Of all the risks we have discussed today or any others you can think of, what is the most worrisome to you? We compared these two points in the interview to examine both first impressions after presentation of the approach and the effect of the in depth discussion and presentation of scenarios on these attitudes. Finally, we asked interviewees if the benefits of HIV ME outweigh the risks.

\section{Analysis of interview data}

Interviews were audio recorded and transcribed. In addition, CS prepared brief summaries from her field notes. Transcripts were coded in Atlas.ti. ${ }^{15}$ with a grounded theory approach, ${ }^{16-18}$ using a combination of question-specific codes and codes developed from the themes that emerged across interviews. Codes were identified and codified by CS and MK. Transcripts were coded by either CS or MK. The coded content discussed in this article was again reviewed by $\mathrm{CS}$ at the time of writing.

\section{Public forum questionnaires}

The authors began this project with the conviction that determining ethical standards for the use of HIV ME should include people who are not engaged in developing the technology. To this end, the project included a set of public forums in which HIV ME was introduced and discussed. The first of these forums was held during the development of the interview guide (two weeks before the initial interview) and informed our approach to presenting the benefits and risks of the technique. Alongside our qualitative findings, we present some of what we learned from informal questionnaires given to attendees of the public forum on HIV ME. These questionnaires captured opinions of more individuals about the risks and benefits of HIV ME, but are not intended as a definitive measure of the general public's opinion.

The Exploring Ethics forum is a regular series organized by San Diego's Reuben H. Fleet Science Center and the Center for Ethics in Science and Technology. The forum on HIV Molecular Epidemiology began with a 20-minute presentation about HIV ME describing the method, suggested applications, and possible risks of the approach. Informal questionnaires were distributed to attendees after the talk, and before the question and answer portion of the program. All answers were collected anonymously and a waiver of consent for these questionnaires was obtained from the UC San Diego Human Research Protections program. One questionnaire in which the respondent indicated he or she was less than 18 years old was discarded.

The lecture presented the primary benefits of HIV ME as related to public health and research, and the questionnaire asked attendees to respond to these benefits and potential risks. Attendees were asked to evaluate four potentially positive outcomes of HIV $\mathrm{ME}$ and four potentially negative outcomes, or risks of HIV, and rate the strength of these potential benefits and risks for populations studied with HIV ME. They were also asked to respond to the question if participants are adequately informed of risks and agree to participate, should [HIV ME research] be done?

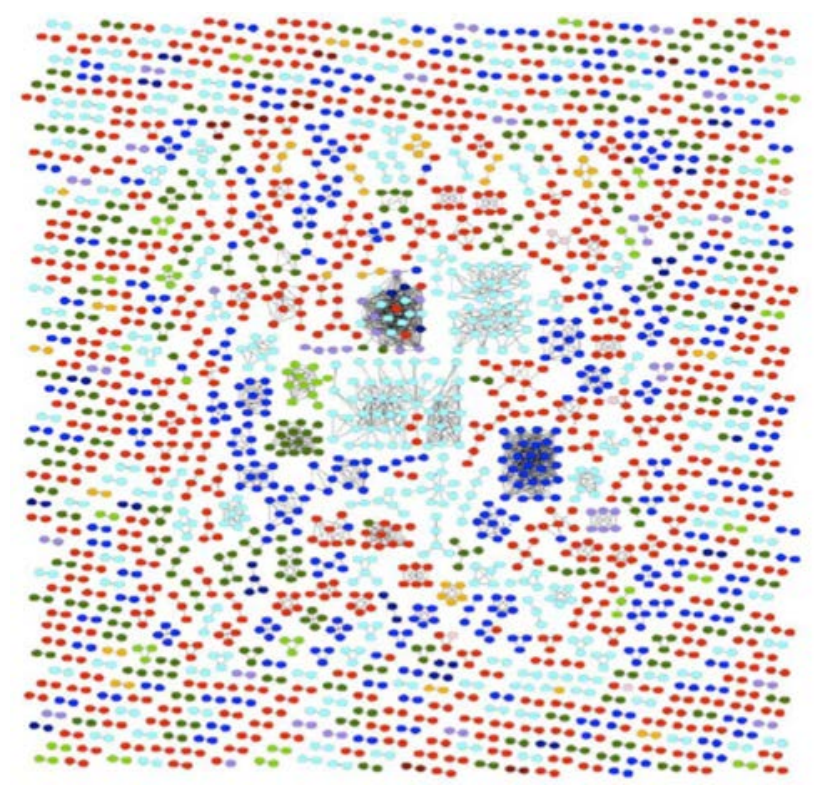

Figure 1. Sample Network Diagram used in interview to illustrate how molecular epidemiology works. Each oval represents an HIV infected individual. Grey lines represent putative linkages between individuals with genetically similar HIV sequences. Colours may represent any demographic variable, for example, zip code, HIV risk factor or ethnicity. 


\section{Results}

\section{Support for molecular epidemiology}

There was general support for molecular epidemiology among the public forum attendees. After rating the benefits and risks of HIV ME, 50 of 53 attendees answered that the research should be done because the potential benefits to public health outweigh risks to individual participants. One attendee was unsure and two failed to answer this item.

Most interviewees also expressed support for the use of HIV ME. At the conclusion of each interview, when respondents were asked if the benefits of HIV ME outweigh the risks, all said yes. Three-quarters of respondents answered with an unqualified, emphatic, or even enthusiastic, yes, to the concluding question. The other quarter of respondents gave a positive answer with qualifications, such as It's very necessary, just as long as parameters are set in place and they're kept (\#161), or, With proper protections in place, the benefits outweigh the risks (\#386). Figure 2 shows the two types of answers by subject group. Of the 10 answers with caveats, 1 came from an HIV positive respondent, 2 from HIV at risk respondents, 3 from medical professionals, and 4 from nonmedical professionals.

\section{Benefits and risks}

Questionnaire responses collected at the public forum suggested general agreement about benefits of HIV ME, but more diversity in responses about risks. The interviews, then, gave us a chance to explore the types of risks interviewees could anticipate and how they responded to these risks.

Comparing basic descriptive statistics of the questionnaire answers illustrates the trend among the public forum attendees. Table 2 presents the means and standard deviations for the ratings of each listed benefit and Table 3 presents the means and standard deviations for the rating of each listed risk. The listed benefits received a mean rating of 4.6 ( 5 being very valuable), while the risks listed on the questionnaire received a mean rating of 3.0 (5 being very worrisome). Comparison of the standard deviations show that the distribution of answers regarding benefits was far more uniform than that of the risks. Figures 3 and 4 show the frequencies of ratings for each type of benefit and risk. Nearly all questionnaire respondents rated the benefits neutrally or positively, while responses to the risks were more evenly distributed between not concerned and very concerned, creating a near neutral mean.

Most interviewees were asked specifically about risks and benefits twice; first after the initial explanation of HIV ME and the again at the conclusion of the interview. The range of risks voiced

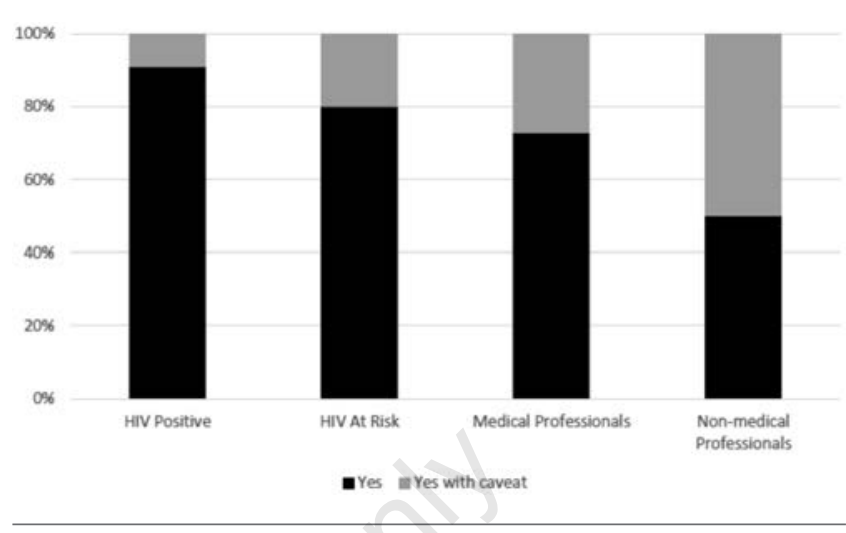

Figure 2. Responses by subject group to final interview question: Given the benefits and risks we just talked about, do you think the benefits outweigh the risks?

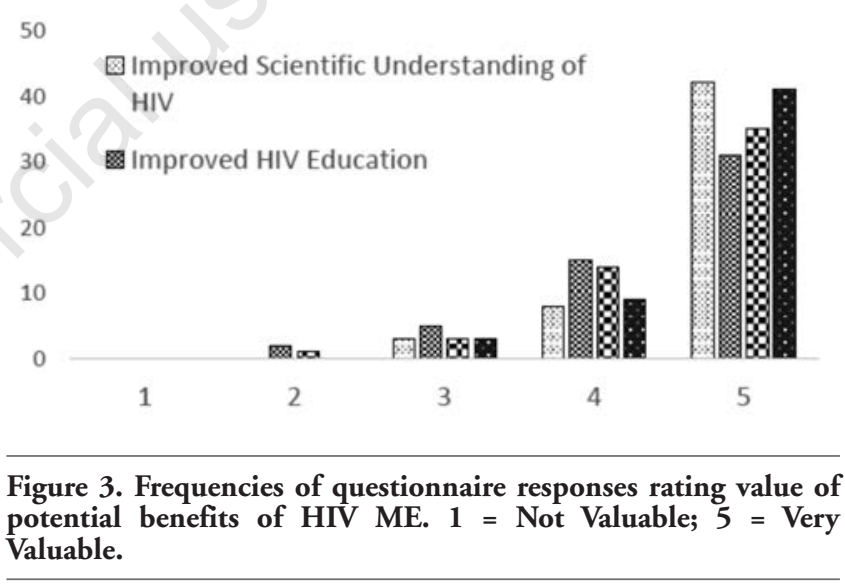

Table 2. Mean ratings of the value of benefits of HIV transmission research by attendees to public forum.

\begin{tabular}{|c|c|c|}
\hline $\begin{array}{l}\text { In your opinion, how valuable are the following potential benefits of HIV Network Research? } \\
\text { (1=Not Valuable, } 5=\text { Very Valuable) }\end{array}$ & Mean & $\begin{array}{l}\text { Std. } \\
\text { Deviation }\end{array}$ \\
\hline Improved understanding of HIV transmission & 4.7 & 0.56 \\
\hline Improved public education about HIV transmission & 4.4 & 0.82 \\
\hline Interventions (testing or education) tailored to a particular group & 4.6 & 0.69 \\
\hline Treatment of individuals identified as high-risk & 4.7 & 0.57 \\
\hline
\end{tabular}

Table 3. Mean ratings of concern over risks of HIV transmission research by attendees to public forum.

\begin{tabular}{|c|c|c|}
\hline $\begin{array}{l}\text { How concerned are you about the following potential risks of HIV Network Research? } \\
\text { (1=Not Concerned, } 5=\text { Very Concerned) }\end{array}$ & Mean & $\begin{array}{l}\text { Std. } \\
\text { Deviation }\end{array}$ \\
\hline Personal health information gets lost or stolen & 3.0 & 1.18 \\
\hline Personal health information becomes publicly accessible & 3.4 & 1.35 \\
\hline Easier for others to guess an individual's HIV status & 3.0 & 1.29 \\
\hline Research reveals who may have been the source of an HIV infection & 2.7 & 1.43 \\
\hline
\end{tabular}


early in the interviews suggest, like the questionnaire answers, how little consensus there may be among the casually informed regarding the risks of HIV ME.

\section{Initial concerns}

Most interviewees identified potential risks or concerns at the start of the interview, whether spontaneously or prompted. The issues they raised related to a) privacy, b) public response to HIV ME, c) potential setbacks that might be faced by the research team (usually in recruiting subjects), and d) other concerns such as psychological damage to subjects. Each of these categories is described below. Figure 5 illustrates how these categories of concern were distributed across groups.

\section{Privacy}

About one quarter of interviewees anticipated some privacy concern early in the interview, either saying that they would be concerned or that they felt others would be concerned. Some respondents spoke about potential dangers of being able to single out individuals based on their position in an HIV transmission network. Others raised the possibility of research subjects finding out new information about each other. A physician mentioned that inadvertent disclosure of a particular set of relationships could raise questions of infidelity, or un-closeting someone who didn't wish that, that would be a potential issue. (\#469) An HIV+ interviewee said, It's more of a privacy issue for myself as well. I honestly wouldn't want to know who gave it to me, how I got it or whatnot, just due to the fact that even if I did know, there's nothing I could really do about it no more. (\#183) An HIV at risk respondent mused:

If [subjects] talk amongst themselves they could very well conclude that maybe they're all part of a same group or that others amongst themselves - if they are in fact friends that someone else is infected or that there's a common source, or we could get into something, infidelity, where there's maybe just the one cheating boyfriend who's bouncing around... So that's one risk I suppose, losing anonymity within the research program itself. (\#215)

A non-medical professional who handles epidemiological data talked about how, when the population is large enough, it would be difficult to identify any one subject, but when reporting on a smaller or more rural population, researchers need to be more careful. This professional explained that if their team is examining a raceethnicity that's unusual for that area or a risk group that's less usual for that area, some of those things set off alarms for us. (\#350) This professional interviewee drew a parallel between HIV $\mathrm{ME}$ and other epidemiology studies that may divide the population in question into small enough groups that individual identity might be guessed.

\section{Public response}

Concerns about public response to HIV ME were primarily about the findings being used to promote anti-gay sentiment, potentially threatening hard-won gay rights. One HIV positive respondent worried, Probably going to have a couple, three hate crimes or... tagging the walls about graffiti and bad stuff about people that are gay (\#141). An HIV at risk respondent said, I think if that information got out, I think that would just add fuel to the fire in certain ways... This epidemiology that you'll be able to do will really bring a light to how promiscuous at least gay men are and bisexual men too that are married to women. (\#212) When asked, at that early point in the interview, if he thought the risks outweighed the benefits, this respondent said, You know the sad thing is, yeah, I think you're about 20 years too soon. (\#212) A non-medical professional echoed this concern, I can imagine it coming up in political campaigns. In a time where the Supreme Court just ruled on marriage equality, someone looking at this and using it as evidence that this whole gay community is out of control. (\#348)

\section{Research setbacks}

Some respondents saw issues of privacy primarily as a problem for the integrity of the research, rather than a true risk to subjects. While these answers focused on privacy, they did not indicate that the respondent understood the particular privacy concerns that are specific to ME. These answers often focused on how the

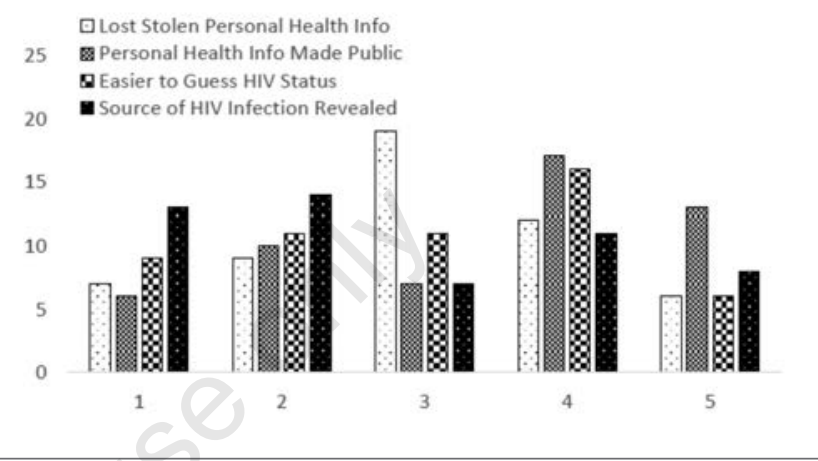

Figure 4. Frequency of questionnaire responses rating concern over potential risks of HIV ME. 1 = Not Concerned; 5 = Very Concerned.

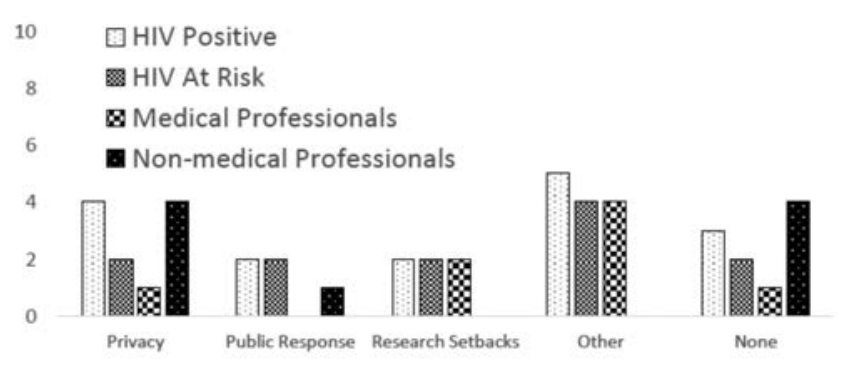

Figure 5. Concerns raised early in the interview, after the first explanation of molecular epidemiology, by subject group.

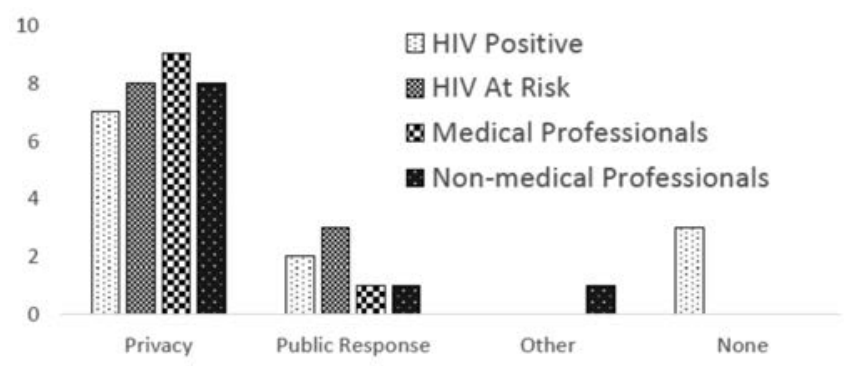

Figure 6. Concerns identified as most worrisome at conclusion of interview, by subject group. 
privacy concerns of potential subjects might make it difficult to recruit for the study or skew findings. An HIV at risk respondent said, Someone who likes to hold on to their social status, who likes to be flirted with, who likes to hook up with guys, they will keep that information private, so they won't contribute to this. (\#252) Another HIV at risk respondent said, the only downside would be, are people withholding honest answers because of their fear of privacy?. (\#263) A physician spoke at length about the problems of recruiting in rural communities, concluding, That is where you are going to get biases in ... that you are probably, [recruiting] people who are at least comfortable with their sexuality or/and their HIV status. (\#457)

\section{Other concerns}

Some initial concerns focused on possible dangers to subjects. For example, an HIV positive respondent wondered if the researchers would be held responsible if a person doesn't take the information in a mentally well-adjusted way, and then they go and commit a crime. (\#145) Another HIV positive respondent was concerned that simply being in a study related to HIV shortly after being diagnosed "would be scary" because it would push them to come to terms that they are HIV-positive (\#105). Other interviewees worried about the potential of HIV ME to identify who infected whom. Such concerns focused on how fear of accusations and legal action may create resistance to the research.

\section{Final concerns}

Although initial concerns were diverse, by the end of the interview most felt that privacy was the most worrisome risk related to the technique. Figure 6 shows the final concerns raised by each subject group. Answers were not mutually exclusive as some respondents raised more than one concern or raised a concern that was mitigated by their trust in the research process. At the end, nearly three-quarters of interviewees discussed some aspect of privacy. Some spoke generally about a loss of confidentiality, while others described more specifically the consequences of such a breach, usually either focusing on interpersonal problems arising from one's HIV status being disclosed, or on possible sources of discrimination based on disclosed HIV status. Only 6 of the individuals who concluded with privacy concerns had named privacy as a potential risk at the beginning of the interview. Seven respondents concluded the interview citing concerns about the public response to HIV ME as most worrisome, including 4 respondents who had anticipated privacy concerns at the outset. Finally, three HIV positive respondents said they had no concerns while acknowledging that privacy might be a concern for others.

\section{Common misunderstandings}

While there was overwhelming support for molecular epidemiology of HIV, there were some common misunderstandings about HIV ME among respondents. Both professional and lay respondents demonstrated similar confusion with some specific aspects of HIV ME.

One set of misunderstandings stemmed from difficulty in grasping the rapid mutation and evolution of HIV that provides the phylogenetic signal to infer relationships between sequences. This often resulted in a failure to understand why the sequences and their relationships could potentially generate genetically identifiable information. Such confusion made it difficult for some respondents to appreciate the sensitivity of the information created by molecular epidemiology, specifically that an individual could be identified as part of an outbreak.

Another set of misunderstandings cantered on the interpreta- tion of the sample network diagram (Figure 1). Many had difficulty understanding what was represented by the grey lines (edges) connecting two individuals (nodes). The interviewer always explained that "the dots that are connected with lines are viruses that are closely related. This means that they are less than $1.5 \%$ genetically different. Unrelated infections could be up to $20 \%$ different." Nevertheless, many respondents persisted in assuming that these lines indicated a confirmed or reported encounter between the individuals. One subject focused on the colours of the nodes, which represented geographic location, and did not seem to appreciate that the diagram showed more than this information. Other subjects incorrectly inferred directionality of infection despite efforts to underscore that the diagram did not show this, and that, furthermore, all links were only putative. In some cases, misreading of the diagram appeared to convince interviewees that the technique has more power than it does (i.e. it could show who gave me $H I V)$ or support a sense of complacency (i.e. don't we already know this?).

Some respondents appeared to have the impression that HIV ME could determine if a specific undiagnosed individual had been exposed to or acquired HIV. For example, an HIV positive respondent said, So, for me, [the benefit is] to call a person and to let them know what they have and all that in a way it's to save their lives. Because if you don't, down the road, you know, they might get sick to a point where there's nothing you're gonna be able to do (\#172). This quote describes a scenario where researchers are able to contact a particular person who has not been tested for HIV to let them know that they are infected. Similarly, another respondent felt that the only benefit of the technique would be to discover people who are high risk and HIV negative. These misunderstandings overestimate the power of the technique. HIV ME may predict the probability of exposure within certain groups, but it cannot determine exposure or infection of specific individuals who might then be personally contacted.

\section{Discussion}

Among interviewees and public forum attendees, there was near unanimous support for HIV ME research. However, the interviews make it clear that this support is conditional. Interviewees expressed concerns about breaches of privacy and how public interpretation of HIV ME studies could lead to increased anti-gay sentiment. Some interviewees were careful to specify that the research should be done as long as proper protections are put in place.

Concerns about molecular epidemiology were not immediately apparent to many of the participants. Some of the interviewees who did raise potential problems did so only with direct prompting to do so. Some spoke of worries that others might have, but emphasized that they were not personally concerned. Many interviewees voiced more concerns about risks during the concluding questions of the interview than they did early on. Only seven interviewees described, at the outset of the interview, the same issue they identified as most worrisome at the conclusion. This suggests that interviewees were not immediately able to identify risks they were most concerned about and had to be walked through potential scenarios before they could identify worrisome risks. Of course, by the end of the interview many responded directly to the privacy concerns rehearsed by the structure of the interview. Compared to those who anticipated privacy concerns at the start of the interview, many more interviewees identified privacy issues as worrisome at the end of the interview. 
While over the course of the interviews most subjects were able to appreciate the risks of HIV ME research, all interviewees said the benefits of HIV ME research outweighed the risks and that the research in this area should continue. Similarly, the questionnaire respondents gave mostly positive ratings for benefits and neutral ratings for risks, suggesting that people responded more to potential benefits than possible risks. It is difficult to assess the capacity of the interviewees to fully understand molecular epidemiology. Even in the interviews with medical and non-medical professionals, there were moments that suggested confusion about the potential of HIV ME. The misunderstandings identified here were not demonstrated clearly in the majority of interviews, but other interviewees could have harboured similar but unstated misconceptions. Indeed, some of these misunderstandings only came to light with the interviewees who were sufficiently sophisticated to articulate clear questions. Unchallenged assumptions would not have been so easy to capture. The misunderstandings we have identified suggest a tendency to either over- or under-estimate the potential of HIV ME to uncover sensitive information. Those who fail to understand what is novel about the technique will not appreciate the unique privacy challenges it presents. On the other hand, those who attribute more accuracy or predictive power to HIV ME than is possible may become overly worried about unlikely or impossible scenarios. In either case, these misunderstandings threaten the possibility of obtaining informed consent.

New and rapidly developing technologies like molecular epidemiology are difficult to explain to a broad audience, especially when the developers and early adopters themselves do not fully understand the implications of their work. Confusion, misunderstandings, and blind spots are understandable, but this does not excuse researchers from the task of explaining the approach and the unknowns to potential subjects. However, the discussions we had in the context of these interviews were responsive to each interviewee, often took significant time, and yet did not always result in an understanding that could be construed as informed. This presents a challenge for researchers who want to inform their subjects but will not have time for such in-depth discussions. This is further complicated in ME research where the degree of risk and benefit is not well understood.

\section{Limitations}

In this article, we focus on questions of informed consent and risk to research subjects, both traditionally understood as issues in research ethics. However, studies using HIV ME often aim to refine or develop new strategies for HIV prevention in the context of public health. When describing the risks and benefits of HIV ME to lay persons, we found it difficult to restrict discussion to research applications only. Our explanation of the technique depended on illustrating the power of the technique with examples of potential public health interventions based on HIV ME. While much of the interview guide focused on HIV ME in a research setting, specific hypotheticals were included involving use of HIV ME analyses by public health departments and attorneys. In this paper, we report on responses to questions focused on the research setting. At the end of the interviews, after discussing both research and public health contexts, interviewees were specifically asked to focus on the research context, but it is not possible to know if interviewees were able to maintain this distinction when thinking about their concerns. Similarly, the questionnaire prompts given to forum participants were intended to refer to HIV ME carried out by researchers rather than public health officials, but the question explicitly asked respondents to weigh the benefits to public health when considering their answer. Because of these ambiguities, is not possible to know if forum participants maintained a clear dis- tinction between HIV ME used in a research setting and possible use in a public health setting when answering this question.

Like all qualitative studies, this work can identify a range of concerns but cannot reliably associate those concerns with large groups of people. The specific composition of our participant group must be taken into consideration. This study focuses on the points of view of potential research subjects and the professionals who work with them. Most interviewees were already invested in HIV research and are of particular interest and importance as they stand to be most affected by HIV ME. As many of the HIV positive and at risk interviewees were concurrently or had in the past participated in medical research related to HIV, they may have been more likely to discount risks to their privacy compared to others who hesitate to serve as research subjects. The thoughts and opinions of people unwilling to be subjects obviously could not be captured and the public acceptance of HIV ME may be less supportive than what we encountered.

Likewise, we do not have sufficient data to know if certain types of responses to HIV ME might be more or less prevalent in subgroups. We do not have sufficient numbers to state, for example, whether women feel differently than men, or MSM have different concerns than injection drug users. Perhaps our work will inspire future studies on such topics.

The data collected from attendees to the public lecture were exploratory and cannot be interpreted as reflecting general public opinion. The group was relatively small and self-selected and had little incentive to think deeply about their answers. The setting and the short format of the questionnaire prevented collection of potentially identifying information regarding background and demographics.

\section{Conclusions}

Overall, we found wide support for using molecular epidemiology to study HIV epidemics and transmission, but with concerns about potential risks to personal privacy and provocation of antigay sentiment. Unfortunately, both support for and concerns about this approach may be based in part on flawed understandings of molecular epidemiology.

In our interviews, only a handful of interviewees understood the privacy implications after the initial description of HIV ME, indicating that the risks of HIV ME may be difficult for both lay and professional individuals to grasp without deep knowledge of the technique. This raises questions about the sufficiency of standard informed consent procedures for HIV ME research; if research subjects and the professionals they work with cannot easily appreciate the sensitivity of the information generated by HIV $\mathrm{ME}$, and the risks of inferential disclosure, they cannot be said to have offered or made an informed choice that protects the interests of subjects.

Because of the potential for severe legal and personal consequences, HIV ME dramatizes general shortcomings in our approach to privacy in public health research. Any study that too exactly describes individuals from a small group or links such descriptions to genetic information is vulnerable to the triangulation techniques identified by Gymrek et al. ${ }^{10}$ Yet there do not appear to be sufficient guidelines for reporting in studies with small numbers of participants or studies that link genetic profiles to sets of identifiable traits. Techniques that allow formal analysis of privacy risk could play an important role in protecting the sensitive information generated by research, but developing and deploying these modern methods of privacy protection will require 
stakeholders to decide how much individual privacy might be risked in the name of public health.

The future of HIV ME as a tool for prevention will depend on the ability of researchers and public health staff to perform their work while safeguarding the privacy of their constituents. The discussion here focused on the research setting, but issues of confidentiality, trust, and public perception of HIV ME will be even more pressing should the technique be employed by public health agencies. Researchers, leaders in public health, and legislators will need to work together to both protect the information they gather and improve the public's understanding of these techniques. As this technique is taken up by public health agencies, it will also be necessary to develop policies, procedures and best practices to ensure the privacy of the general public is also sufficiently protected. This early research on perceptions and attitudes of stakeholders in HIV ME research points to how crucial and challenging such work will be.

Correspondence: Sanjay Mehta, University of California San Diego, $200 \mathrm{~W}$. Arbor Drive \#8208, San Diego, CA 92103-8208, USA.

Tel.: +1.619.543.8080 - Fax: +1.619.543.5066.

E-mail: srmehta@ucsd.edu

Funding: This work was supported by funding from the National Institutes of Health AI093163 (SRM), AI106039 (SL), Al108351 (SL, MK, SAV), MH100974 (SL, SRM, SAV), UL1 TR001442 (MK), MH081482 (Interdisciplinary Research Fellowship in NeuroAIDS, MH), P30 AI036214 (MH, SRM, SL), and P50 DA026306 (MH).

Key words: HIV; molecular epidemiology; qualitative interviews; privacy; research ethics

Contributions: SRM, SAV, MK and SL designed the study; CS performed the interviews; MK organized the forums; CS, SRM, SAV, MH, MK and SL analyzed the data; CS and SRM wrote the manuscript; and CS, SRM, SAV, MH, MK, and SL all edited and approved the final manuscript. MK and SL contributed equally to this manuscript.

Conflict of interest: the authors declare no potential conflict of interest.

Received for publication:11 May 2017.

Accepted for publication: 4 September 2017.

(C) Copyright C. Schairer et al., 2017

Licensee PAGEPress, Italy

Journal of Public Health Research 2017;6:992

doi:10.4081/jphr.2017.992

This work is licensed under a Creative Commons Attribution NonCommercial 4.0 License (CC BY-NC 4.0).

\section{References}

1. Rosenberg ES, Grey JA, Sanchez TH, Sullivan PS. Rates of prevalent HIV infection, prevalent diagnoses, and new diagnoses among men who have sex with men in US States, metropolitan statistical areas, and counties, 2012-2013. JMIR Public Health Surveill. 2016;2:e22.

2. Bonacci RA, Holtgrave DR. Evaluating the Impact of the US National HIV/AIDS Strategy, 2010-2015. AIDS Behav 2016;20:1383-9.

3. Holmes EC. Error thresholds and the constraints to RNA virus evolution. Trends Microbiol 2003;11:543-6.

4. Little SJ, Kosakovsky Pond SL, Anderson CM, et al. Using HIV networks to inform real time prevention interventions. PLoS One 2014;9:e98443.

5. Dennis AM, Hue S, Hurt CB, et al. Phylogenetic insights into regional HIV transmission. AIDS 2012;26:1813-22.

6. Bello G, Eyer-Silva WA, Couto-Fernandez JC, et al. Demographic history of HIV-1 subtypes B and F in Brazil. Infect Genet Evol 2007;7:263-70.

7. Poon AF, Gustafson R, Daly P, et al. Near real-time monitoring of HIV transmission hotspots from routine HIV genotyping: an implementation case study. Lancet HIV 2016;3:e231-8.

8. Brooks JI, Sandstrom PA. The power and pitfalls of HIV phylogenetics in public health. Can J Public Health 2013;104:e348-50.

9. Pillay D, Rambaut A, Geretti AM, Brown AJ. HIV phylogenetics. BMJ. 2007;335:460-1.

10. Gymrek M, McGuire AL, Golan D, et al. Identifying personal genomes by surname inference. Science 2013;339:321-4.

11. Mehta SR, Vinterbo SA, Little SJ. Ensuring privacy in the study of pathogen genetics. Lancet Infect Dis 2014;14:773-7.

12. Hecht FM, Wolf LE, Lo B. Lessons from an HIV transmission pair. J Infect Dis 2007;195:1239-41.

13. Lehman JS, Carr MH, Nichol AJ, et al. Prevalence and public health implications of state laws that criminalize potential HIV exposure in the United States. AIDS Behav 2014;18:997-1006.

14. Finitsis DJ, Stall RD, Friedman SR. Theory, analysis, social justice, and criminalizing HIV transmission: a commentary on Lehman and colleagues (2014). AIDS Behav 2014;18:100710 .

15. ATLAS.ti 7, Qualitative Data Analysis. 2017.

16. Glaser BG, Anselm AL. The discovery of grounded theory: strategies for qualitative research. Chicago: Aldine; 1967.

17. Glaser BG. Basics of grounded thery analysis. Mill Valley, CA: Sociology Press; 1992.

18. Charmaz K. Constructing grounded theory: a practical guide through qualitative analysis. Thousand Oaks, CA: Sage Publications; 2000. 